Military Technical College Kobry El-Kobbah Cairo, Egypt

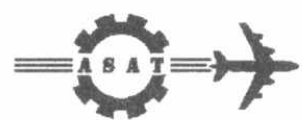

$10^{\text {th }}$ International Conference

On Aerospace Sciences\&

Aviation Technology

\title{
Monitoring Depth of Anaesthesia using Fuzzy Logic System
}

\section{A.Elkfafi*}

\begin{abstract}
The depth of anaesthesia (DOA) achieved is a result of the concentration of the anaesthetic drugs in the brain. Until recent years, there has been no simple, accurate and objective method for assessing the DOA or alerting the anaesthetist to a state of awareness. The monitoring of DOA is complex, and dependent on many factors, which vary between patients and operating procedures. A method that can be adapted for handling complex and inexact knowledge (e.g. DOA) within a computer program is fuzzy set theory. This paradigm seems to be specially suitable for medical processes, since it depends upon expert experiences which are not precisely quantifiable, such as patients' subjective sensations, interpretation of clinical signs and effects of instrumental accuracy.

In this paper, two significant extracted features from the processed auditory evoked response (AER) signal, describing the changes in amplitudes and latencies of MLAER waves, have been merged together using fuzzy logic to create a reliable index for DOA every 30 seconds.

\section{KEY WORDS}

Depth of Anaesthesia (DOA), Mid Latency Auditory Evoked Response (MLAER),

Evoked Potential (EP), Fuzzy Logic System (FLS), Averaged Phase of MLAER signal in $\mathrm{S}$ - Domain (Ph), Averaged Magnitude of MLAER signal in S - Domain (Mag), Feature Extraction (FE), Fuzzy Logic Modeling (FLM), Digital Signal Processing (DSP), Auditory Evoked Response (AER).
\end{abstract}

\footnotetext{
* Egyptian Armed Forces.
} 


\section{I) introduction}

A sensory evoked potential (EP) is the electrical activity that's elected in a nervous pathway by a sensory stimulus [Kakigi, 1994]. The auditory stimulus (click or tone) can be used for electing the AER. The somatosensory stimulus (electric pulse) can be used for electing the somatosensory evoked response (SER). The visual stimulus (flashing light) can be used for electing the visual evoked response (VER). The signal used for measuring DOA should show graded changes with anaesthetic concentration, similar changes for different agent, appropriate changes with surgical event, and indicate awareness. The above criteria have been used to examine the suitability of a various evoked potential for measuring DOA, having validated the MLAER as a measure of anaesthetic depth [Thronton. C., 1991].

The measuring of DOA is very important because it helps the anaesthetists to monitor the anaesthetic state of the patient from the start of giving the anaesthetic drugs up to the patient awakeness and helping him to control the required amounts of the anaesthetic drugs during the surgery [Munglani, 1994]. There is no direct method to judge the DOA. Monitoring of DOA is complex, and dependent on many factors which vary between patients and operating procedures. However, anaeshtesits in operating theater can monitor and manage patient very well, based on their experience and knowledge. Much of human problem solving and inference is uncertain, inexact and partial, i.e. it is fuzzy. in many circumstances where decisions have to be made, the facts are far from precise. A method that can be adapted for handling inexact facts using a computer program is the fuzzy set theory. This paradigm seems to be especially suitable for medical process, i.e. Anaesthesia. The fuzzy logic technique is used to model the DOA. Therefore, there is a need for a reliable and accurate means of monitoring anaesthetic depth.

Fuzzy logic theory is a mathematical theory, which caters for one aspect of uncertainty. Fuzziness is the ambiguity that can be found in the definition of a concept of the meaning of a word, for example; the uncertainty in expressions likes "DOA" can be called fuzziness [Zadah, 1973]. The basic configuration of a simple fuzzy logic system is shown in Fig. (1), and comprises four principal components; a fuzzification interface, a knowiedge base, a fuzzy inference engine and a defuzzification interface [Lee, 1990a].

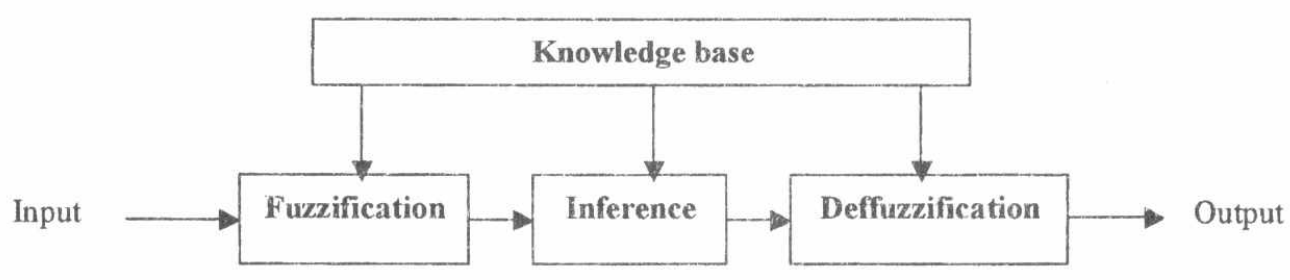

Fig. 1. Basic Configuration of fuzzy logic modeling (FLM) 


\section{II) The Proposed System for Monitoring Depth of Anaesthesia}

The structure of the system for monitoring depth of anaesthesia is shown in Fig. (2). In this research work, the system estimates the depth of anaesthesia from off- line AER data. A digital signal processing (DSP) technique was carried out to smooth the MLAER and improve the signal to noise ratio $(\mathrm{S} / \mathrm{N})$ [M. Elkfafi., et al., 2003, (in press)]. Furthermore, feature extraction (FE) was implemented to extract the factors (i.e., Mag. and $\mathrm{Ph}$.) describing the changes in magnitude and phase of MLAER waves every 30 seconds [M. Elkfafi., et al., 2003, (in press)]. In this paper, the above two factors have been merged together using fuzzy logic to create a reliable index for DOA every 30 seconds. The system rules can be taken from anaesthetists' experience, in which case we apply a simple fuzzy modeling system as shown in Fig. (2) to model the depth of anaesthesia. The merging of phase (Ph) and magnitude (Mag) of MLAER, where Mag is the dominant factor, provides estimates for the DOA. The output of the monitoring system has values from 90 to 500 for the three states of anaesthesia, where Anaesthetic light (AL) is DOA less than 200, Anaesthetic OK (AO) is DOA between 200 and 400 and Anaesthetic Deep (AD) is DOA greater than 400 . The state or $A O$ is divided to three levels: Anaesthetic (max - ok) where DOA is between 320 and 400, Anaesthetic (ok) where DOA is between 260 and 320 , and Anaesthetic ( $\mathrm{min}-\mathrm{ok}$ ) where DOA is between 200 and 260. 


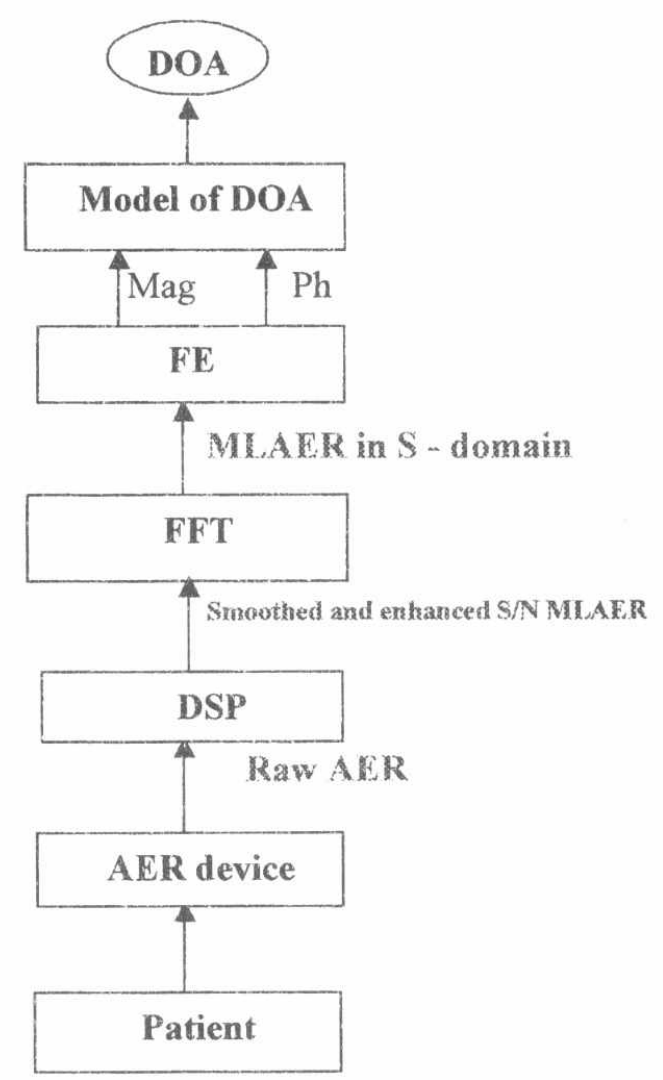

Fig. 2. The structure of the system for monitoring depth of anaesthesia

The proposed system for monitoring DOA shown above has been implemented via computer simulation. The system was tested to interpret the depth of anaesthesia for the whole operation (i.e. induction stage, maintenance stage and recovery stage) in the operating theatre during intravenous anaesthesia using propofol.

\section{III) Rule-base of DOA from anaesthetists' experience}

The two factors Mag. and Ph. are merged together with Mag as the dominant factor to estimate the DOA every 30 seconds. The rules to decide DOA can be expressed verbally. Arising from discussions with anaesthetists, the $\mathrm{Ph}$ and Mag are divided into 
three different ranges i.e. High $(\mathrm{H})$, Medium $(\mathrm{M})$ and Low $(\mathrm{L})$. High means the $\mathrm{Ph}$ and Mag values are higher than normal values and vice versa for Low. Medium means the $\mathrm{Ph}$ and Mag values are in the normal range of values. There are also three states of DOA i.e., Anaesthetic Light (AL), Anaesthetic OK (AO) and Anaesthetic Deep (AD). From discussions with anaesthetists, the rule base to decide DOA is shown in table (1). In this table, the range of each parameter (i.e. Mag, Ph, and DOA) is determined after discussions with anaesthestis and multiplying by empirical factors.

Table 1 Anaesthetist's rule-base for DOA

\begin{tabular}{|c|c|c|c|c|}
\hline \multicolumn{2}{|c|}{ DOA } & \multicolumn{3}{|c|}{ Mag } \\
\hline & & H & $\mathbf{M}$ & $\mathbf{L}$ \\
\hline \multirow{3}{*}{$\mathbf{P h}$} & $\mathrm{H}$ & $\mathrm{AL}$ & $\mathrm{AO}$ & \\
\hline & $\mathbf{M}$ & $\mathrm{AL}$ & $\mathrm{AO}$ & $\mathrm{AD}$ \\
\hline & $\mathbf{L}$ & & $\mathrm{AO}$ & $\mathrm{AD}$ \\
\hline
\end{tabular}

\section{IV) Merging the Ph and Mag to decide the DOA}

After the rule-base for DOA during anaesthesia via propofol has been obtained from discussion with anaesthetists as shown in table (1), the simple fuzzy modeling system can be applied to model the DOA (i.e. complete the remaining undefined rules) as shown previously in Fig (1). If we choose a triangular shape as a membership function and center of area as a defuzzification method, a lookup table can be obtained from table (1) to decide the DOA every 30 seconds during anaesthesia via propofol. After the setting of ranges for the Mag., Ph., and DOA inference was applied to determine the remaining rule. These ranges were expressed by logic values as follows:

$\mathrm{Ph}(\mathrm{H})=-1, \mathrm{Ph}(\mathrm{M})=0, \mathrm{Ph}(\mathrm{L})=+1$,

$\operatorname{Mag}(\mathbf{H})=-1, \operatorname{Mag}(M)=0, \operatorname{Mag}(L)=+1$,

$\operatorname{DOA}(A L)=-1, \operatorname{DOA}(A O)=0, \operatorname{DOA}(A D)=+1$. 
Then, the rules for DOA were defines as:

$\begin{array}{ccc}\text { Mag } & \text { Ph } & \text { DOA } \\ -1 & -1 & -1 \\ 0 & -1 & 0 \\ -1 & 0 & -1 \\ 0 & 0 & 0 \\ 1 & 0 & 1 \\ 0 & 1 & 0 \\ 1 & 1 & 1\end{array}$

Table 2 The logic rules for DOA

\begin{tabular}{|c|c|c|c|c|}
\hline \multicolumn{2}{|c|}{ DOA } & \multicolumn{3}{|c|}{ Mag } \\
\hline & & -1 & 0 & +1 \\
\hline \multirow{3}{*}{$\mathbf{P h}$} & -1 & -1 & 0 & \\
\hline & 0 & -1 & 0 & +1 \\
\hline & +1 & & 0 & +1 \\
\hline
\end{tabular}

Triangular shapes as membership functions, and center of area as a defuzzification method were used to calculate the values of DOA every 30 seconds giving the lookup table for anaesthesia with propofol. The same method has been applied for calculating the rules of DOA for the remaining two rules after calculating the DOA values for all rules, they are formulated in a lookup table as shown in table 3. 
Table 3, Lookup table for DOA

\begin{tabular}{|c|c|c|c|c|}
\hline \multirow{2}{*}{\multicolumn{2}{|c|}{ DOA }} & \multicolumn{3}{|c|}{ Mag } \\
\hline & & H & $\mathbf{M}$ & $\mathbf{L}$ \\
\hline \multirow{3}{*}{$\mathbf{P h}$} & H & -0.60 & -0.17 & 0.33 \\
\hline & $\mathbf{M}$ & -0.50 & 0.00 & 0.50 \\
\hline & $\mathbf{L}$ & -0.33 & 0.17 & 0.60 \\
\hline
\end{tabular}

Defuzzification was applied to obtain the numerical table for DOA, which is shown in table 4, from the lookup table (table 3) by multiplying the values of DOA by an appropriate scaling factor. These numerical values represent the DOA with respect to AER every 30 seconds during intravenous anaesthesia using propofol.

Table 4, numerical table for DOA

\begin{tabular}{|c|c|c|c|c|}
\hline \multirow{2}{*}{\multicolumn{2}{|c|}{ DOA }} & \multicolumn{3}{|c|}{ Mag } \\
\hline & & $\mathbf{H}$ & $\mathbf{M}$ & $\mathbf{L}$ \\
\hline \multirow{3}{*}{$\mathbf{P h}$} & H & 100 & 243 & 410 \\
\hline & $\mathbf{M}$ & 133 & 300 & 467 \\
\hline & $\mathbf{L}$ & 190 & 357 & 500 \\
\hline
\end{tabular}


Fuzzification was applied to obtain the full linguistic table for DOA during intravenous anaesthesia of propofol as shown in table 5 from the numerical table (table 4) by setting the ranges for Mag., Ph., and DOA.

Table 5, Full linguistic table for DOA

\begin{tabular}{|c|c|c|c|c|}
\hline \multirow{2}{*}{\multicolumn{2}{|c|}{ DOA }} & \multicolumn{3}{|c|}{ Mag } \\
\hline & & $\mathbf{H}$ & $\mathbf{M}$ & $\mathbf{L}$ \\
\hline \multirow{3}{*}{$\mathbf{P h}$} & H & $\mathrm{AL}$ & $\mathrm{AO}$ & $\mathrm{AD}$ \\
\hline & $\mathbf{M}$ & $\mathrm{AL}$ & $\mathrm{AO}$ & $A D$ \\
\hline & $\mathbf{L}$ & $\mathrm{AL}$ & $\mathrm{AO}$ & $\mathrm{AD}$ \\
\hline
\end{tabular}

For deciding the DOA values in more detail, three tables for the different anaesthetic states (i.e. $\mathrm{AL}, \mathrm{AO}, \mathrm{AH}$ ) were constructed by dividing each range of $\mathrm{Ph}$ and $\mathrm{Mag}$ (i.e. $\mathrm{H}$, $M, L$ ) into three levels (after discussions with anaesthetists and using appropriate scaling factors). The range High $(\mathrm{H})$ was divided into Big High $(\mathrm{BH})$, Normal High $(\mathrm{NH})$ and Small High (SH). Also, the range Medium (M) was divided to Big Medium (BM), Normal Medium (NM) and Small Medium(SM). Furthermore, the range low (L) was divided to Big Low (BL), Normal Low (NL) and Small Low (SL). The three tables are described as follows: 


\section{(i) For Anaesthetic Light (AL):}

The DOA is less than 200 and its values are shown in more details in table 6 .

Table 6, Numerical table for DOA during AL

\begin{tabular}{|c|c|c|c|c|}
\hline \multirow{2}{*}{\multicolumn{2}{|c|}{$\operatorname{DnA}$}} & \multicolumn{3}{|c|}{ Mag } \\
\hline & & BH & NH & SH \\
\hline \multirow{3}{*}{ Ph } & BH & 90 & 126 & 168 \\
\hline & NH & 98 & 140 & 182 \\
\hline & SH & 112 & 154 & 190 \\
\hline
\end{tabular}

Where the ranges for Mag and $\mathrm{Ph}$ are as follows:

$\mathrm{Ph}(\mathrm{BH})$ : during propofol anaesthesia, the $\mathrm{Ph}$ is greater than 220.

$\mathrm{Ph}(\mathrm{NH})$ : during propofol anaesthesia, the $\mathrm{Ph}$ is between 200 and 220 .

$\mathrm{Ph}$ (SH): during propofol anaesthesia, the $\mathrm{Ph}$ is between 190 and 200.

Mag $(\mathrm{BH})$ : during propofol anaesthesia, the $\sum$ Mag is greater than 2.2 .

Mag (NH): during propofol anaesthesia, the Mag is between 2.1 and 2.2

Mag (SH): during propofol anaesthesia, the Mag is between 2 and 2.1. 
(ii) For Anaesthetic OK (AO):

The DOA is between 200 and 400 and its values are shown in more details in table 7.

Table 7, Numerical table for DOA during AO

\begin{tabular}{|c|c|c|c|c|}
\hline \multicolumn{2}{|c|}{ DOA } & \multicolumn{3}{|c|}{ Mag } \\
\hline & & BM & NM & SM \\
\hline \multirow{3}{*}{$\mathbf{P h}$} & $\mathbf{B M}$ & 200 & 272 & 355 \\
\hline & NM & 217 & 300 & 384 \\
\hline & SM & 245 & 328 & 400 \\
\hline
\end{tabular}

Where the ranges for Mag and $\mathrm{Ph}$ are as follows

$\mathrm{Ph}(\mathrm{BM})$ : during propofol anaesthesia, the $\mathrm{Ph}$ is between 130 and 190. $\mathrm{Ph}(\mathrm{NM})$ : during propofol anaesthesia, the $\mathrm{Ph}$ is between 120 and 130 . $\mathrm{Ph}$ (SM): during propofol anaesthesia, the $\mathrm{Ph}$ is between 0.01 and 120 .

Mag (BM): during propofol anaesthesia, the Mag is between 0.8 and 2 .

Mag (NM): during propofol anaesthesia, the Mag is between 0.6 and 0.8 . Mag (SM): during propofol anaesthesia, the Mag is between 0.1 and 0.6 . 
(iii) For Anaesthetic Deep (AD):

The DOA is greater than 400 and its values are shown in more details in table 8 .

Table 8, Numerical table for DOA during AD

\begin{tabular}{|c|c|c|c|c|}
\hline \multicolumn{2}{|c|}{ DOA } & \multicolumn{3}{|c|}{ Mag } \\
\hline & & BL & NL & SL \\
\hline \multirow{3}{*}{$\mathbf{P h}$} & BL & 420 & 449 & 482 \\
\hline & NL & 427 & 460 & 493 \\
\hline & SL & 438 & 471 & 500 \\
\hline
\end{tabular}

Where the ranges for Mag and $\mathrm{Ph}$ are as follows

$\mathrm{Ph}(\mathrm{BL})$ : during propofol anaesthesia, the $\mathrm{Ph}$ is between 0.001 and 0.01 .

$\mathrm{Ph}(\mathrm{NL})$ :during propofol anaesthesia, the $\mathrm{Ph}$ is between 0.0001 and 0.001 .

$\mathrm{Ph}(\mathrm{SL})$ : during propofol anaesthesia, the $\mathrm{Ph}$ is less than 0.0001 .

Mag(BL):during propofol anaesthesia, the Mag is between 0.075 and 0.1 . $\mathrm{Mag}(\mathrm{NL})$ :during propofol anaesthesia, the Mag is between 0.05 and 0.075 .

Mag (SL): during propofol anaesthesia, the Mag is less than 0.05 .

The above three tables (i.e. table 6, 7, and 8) and the general table 4, were used in conjunction to decide the DOA every 30 seconds according to the following rules:

IF Mag. And Ph. are in the same direction (i.e. both are $H, M$, or L.) THEN the tables 6 , 7 , and 8 are used to decide the DOA.

IF Mag. And Ph. are in opposite direction THEN the general table (4) is used to decide the DOA. 


\section{V) Clinical Results}

The method of monitoring the DOA mentioned above was applied to clinical trials for 21 patients. These cases, who were undergoing body surface (i.e. minor) and abdominal (i.e. major) surgery were studied, with respect to AER during intravenous anaesthesia via propofol in Royal Halamshire hospital Sheffield. Patients with cardiac vascular, hepatic, renal, brain, hearing, or endocrine diseases were excluded.

The off - line results of interpreting the DOA were successful. They are assessed by discussion with anaesthesists and found to be acceptable. The results of two clinical trials are shown in Fig. $(2,3)$ to demonstrate the performance of the numerical index derived from AER (i.e. DOA) as a reliable assessment for monitor the DOA every 30 seconds during anaesthesia with propofol. In this result the DOA increased following induction. It decreased slightly at the intubation. The index mostly remained in the state of anaesthetic (ok) especially in the state of (max - ok), and never went into the state of anaesthetic light or anaesthetic deep. The index returned to the low level when anaesthesia was reversed.

The index showed appropriate changes with the surgical events, graded change with anaesthetic concentration, and indicate awereness (i.e. eye opening). This indicates adequate clinical anaesthesia for all studied cases. Furthermore, this matched the interpretation of anaesthetic depth via anaesthesits' experience using clinical signs and anaesthesits' observations (that none of the cases showed any evidence of awareness during operations.

\section{VI) Conclusions:}

The AER changes seen were compatible with valid DOA measurement. The AER index (i.e. DOA) reflected the changes in amplitudes and latencies of MLAER waves due to drug concentration and surgical stimulation in a way that might be expected in routine anaesthesia practice. So, the DOA has been validated as a reliable assessment of anaesthetic depth during anaesthesia with propofol. Furthermore, it gives an indicator, which can help and wam anaesthestists in operating theater. These successful results have given confidence to perform on - line clinical trials in operating theater in the near future. 


\section{References:}

(1) Kakigi, R., Evoked potentials, Clinical Neurology, 34(12): 1255 - 1257, (1994).

(2) Lee, C. C., Fuzzy logic in control systems: Fuzzy logic controller - Part I, IEEE Trans., Syst. Man Cybern., 20 (2): $404-418$, (1990a).

(3) Munglani, R., Depth of anesthesia, 49(1): 78 - 79, (1994).

(4) M. Elkfafi, S. Elian, M.Gadalla, T.Soliman, Digital signal processing for AER signals during anaesthsia, (2003, in press).

(5) M. Elkfafi, M.Gadalla, S. Elian, T.Soliman, Feature Extraction from MLAER signals during propofol anaesthesia, (2003, in press).

(6) Thronton, C., Evoked potentials in anesthesia, European journal of Anaesthiology, 1991, 8:89-107, (1991).

(7) Zadah, L., A., Outline of a new approach to the analysis of complex a systems and destination process, IEEE Trans. Systems Man Cybernet., SMC - 3, pp. $28-44$, (1973). 


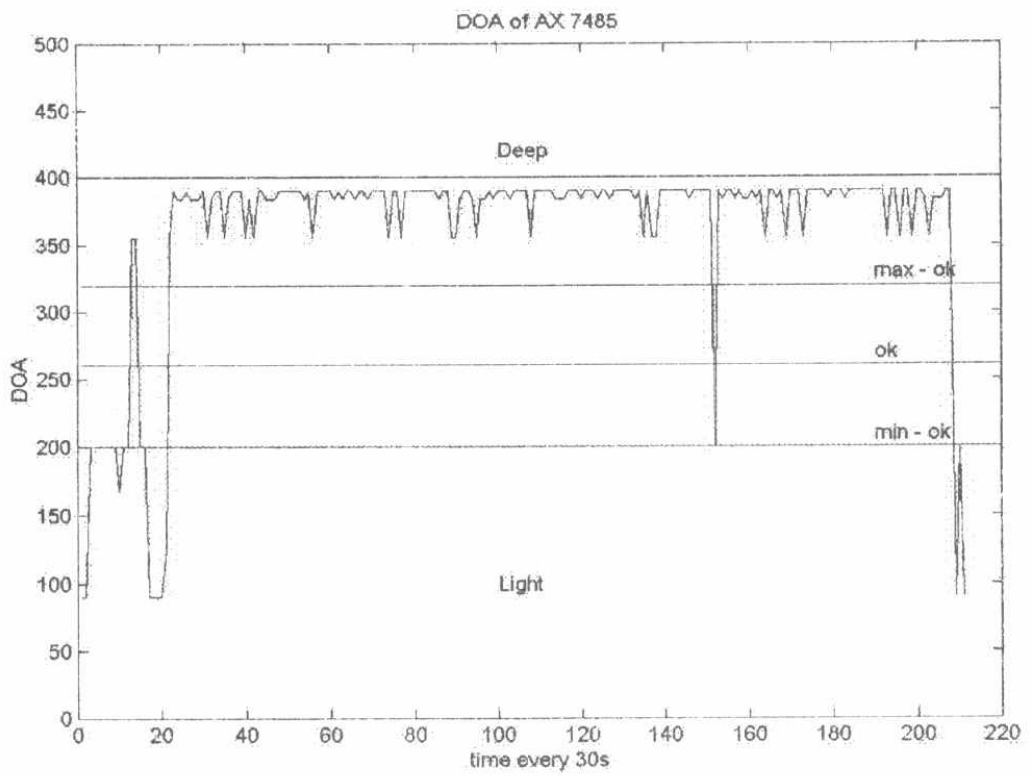

(a)

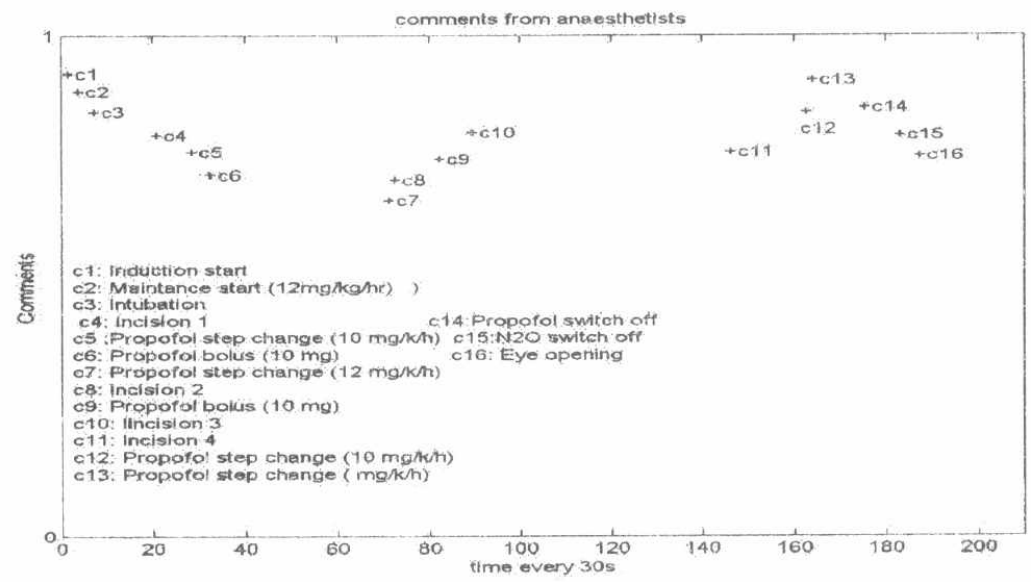

(b)

Fig. 3. The results of interpreting the DOA of patient ax7485. a) DOA; b) Comments from anaesthatists 


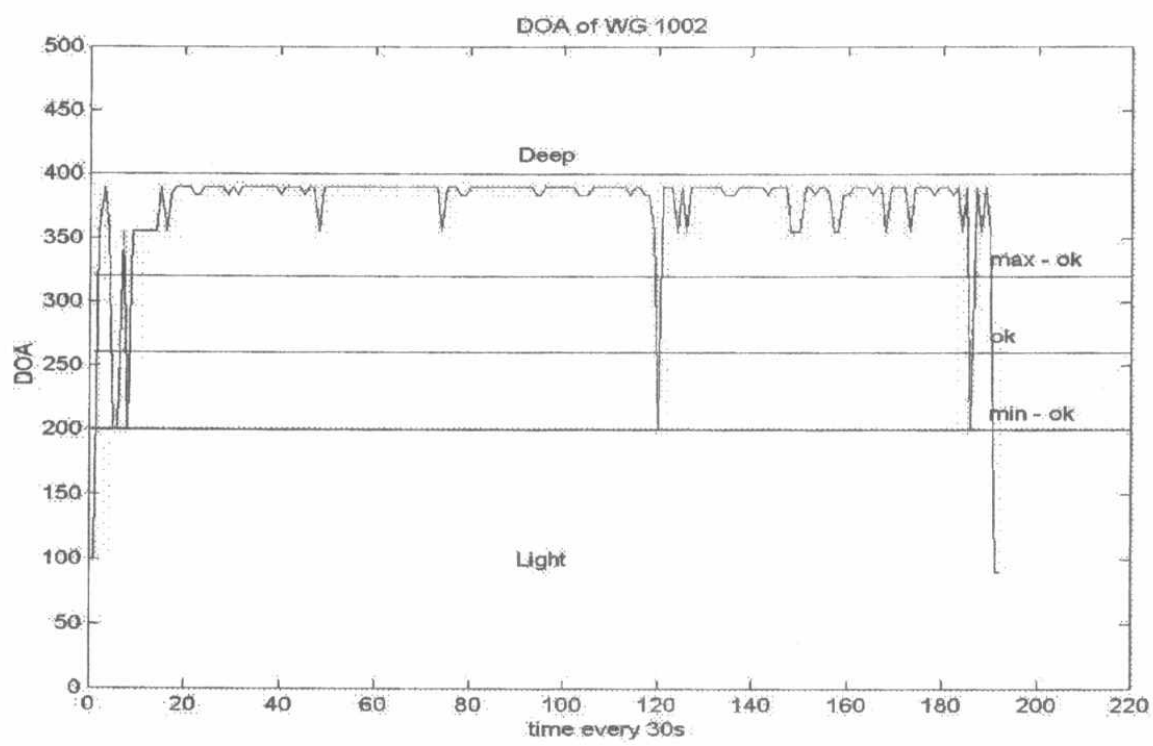

(a)

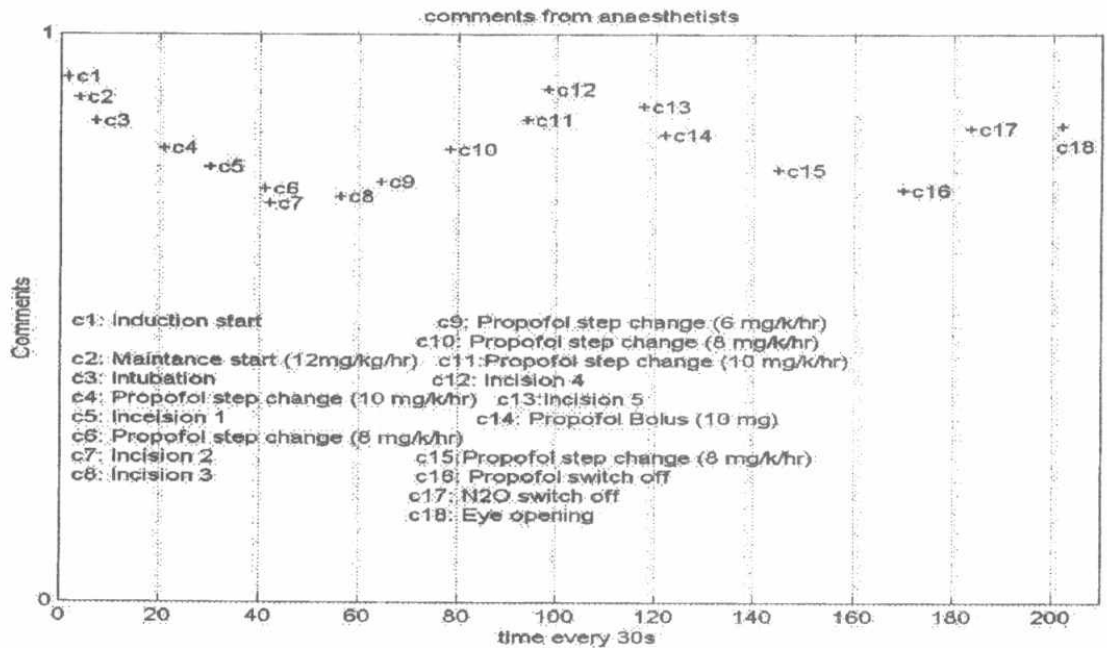

(b)

Fig. 4. The results of interpreting the DOA of patient wg1002. a) DOA; b) Comments from anaesthatists 